\title{
Premium Monovision versus Bilateral Myopic Monovision, Hybrid Monovision and Bilateral Trifocal Implantation: A Comparative Study
}

\author{
Georgios Labiris $\mathbb{D}^{\prime}$, Eirini-Kanella Panagiotopoulou $\mathbb{D}^{\prime}$, Asli Perente', Panagiota Ntonti $\mathbb{D}^{\prime}$, \\ Konstantinos Delibasis $\mathbb{D}^{2}$, loannis Fotiadis ', Aristeidis Konstantinidis $\mathbb{D}^{\prime}$, Doukas Dardabounis $\mathbb{D}^{\prime}$ \\ 'Department of Ophthalmology, University Hospital of Alexandroupolis, Dragana, 68100, Alexandroupolis, Greece; ${ }^{2}$ Department of Computer \\ Science and Biomedical Informatics, University of Thessaly, Lamia, 35I3I, Greece \\ Correspondence: Georgios Labiris, Department of Ophthalmology, University Hospital of Alexandroupolis, Dragana, 68100, Alexandroupolis, \\ Greece, Tel +306977455027, Email labiris@usa.net
}

\begin{abstract}
Purpose: Contemporary monovision techniques use premium intraocular lenses (IOLs), either in both eyes or at least in the nondominant one. Primary objective of this study was to compare the efficacy of premium monovision (implantation of the trifocal diffractive Panoptix IOL in the non-dominant eye and the bifocal hybrid refractive-diffractive Restor IOL in the dominant eye), against bilateral myopic monovision (implantation of the monofocal SN60WF IOL targeting $-0.50 \mathrm{D}$ in the dominant eye and $-1.25 \mathrm{D}$ myopia in the non-dominant one), hybrid monovision (implantation of Panoptix in the non-dominant eye and SN60WF in the dominant eye) and bilateral trifocal implantation (with bilateral Panoptix implantation).

Methods: This is a prospective, comparative, clinic-based trial. Cataract patients populated four study groups: Monovision Group (MoG), Multifocal Lens Group (MfG), Hybrid Monovision Group (HmG) and Premium Monovision Group (PmG). Binocular Uncorrected Distance Visual Acuity (UDVA), Uncorrected Reading Acuity and Critical Print Size at 60cm (UIRA, UICPS) and at $40 \mathrm{~cm}$ (UNRA, UNCPS), contrast sensitivity, vision-related functional impairment, dysphotopsia symptoms and spectacle dependence were evaluated 6 months following the operation of the second eye. A mathematical model was constructed, which calculated the relative efficacy of each surgical intervention.

Results: A total of 120 participants were recruited and populated equally the study groups. Significant improvement of preoperative UDVA was observed in all study groups. No significant differences could be detected in postoperative UDVA and UIRA ( $p=0.24)$ among study groups, while significant differences were noticed in UICPS $(p=0.04)$, UNRA $(p=0.02)$ and UNCPS $(p=0.01)$. Dysphotopic phenomena (glare and shadows) were significantly more in the MfG arm followed by the PmG group ( $\mathrm{p}=0.04$ and $\mathrm{p}=$ 0.02 , respectively), while perceived difficulty and spectacle independence rates were significantly better in PmG group. PmG presented the best overall relative efficacy.
\end{abstract}

Conclusion: All surgical techniques present satisfactory outcomes. Premium monovision seems to demonstrate the best outcomes.

Trial Registration: ClinicalTrials.gov, NCT04618380. Registered 05 November 2020, https://clinicaltrials.gov/ct2/show/ NCT04618380.

Keywords: cataract, presbyopia, monovision, multifocal intraocular lens, premium lens, trifocal lens, bifocal lens, diffractive lens

\section{Background}

It is a truism that presbyopia correction is among the most challenging unmet objectives in ophthalmology. Despite the fact that cataract surgery is the most frequent surgical intervention in medicine, ${ }^{1}$ the postoperative loss of accommodation is yet to be adequately addressed. Different surgical approaches for the correction of iatrogenic and age-related presbyopia have been developed which target the cornea and/or the crystalline lens. ${ }^{2}$ Different technologies have also been introduced, primarily in the ophthalmological lasers and in the intraocular lenses (IOLs) that aim to restore the pre-presbyopic 
functionality of the human eye. ${ }^{3}$ The ultimate goal is a spectacle-free visual capacity that imposes no limits to the person's social, personal, working needs and wants. ${ }^{4-6}$

Pseudophakic monovision is probably the first and most prevalent surgical procedure to address presbyopia. ${ }^{7}$ In traditional pseudophakic monovision, monofocal IOLs are implanted in both eyes; however, the non-dominant eye is intentionally defocused for myopia. ${ }^{3-6,8}$ Myopic defocus of the non-dominant eye ranges from over 2 diopters (D) to less than $1 \mathrm{D}$ (mini or micro monovision). ${ }^{3,4,8,9}$ In bilateral myopic monovision, the dominant eye defocus is targeted to -0.50 $\mathrm{D}$, while the non-dominant one to $-1.25 \mathrm{D} .{ }^{10}$ On the other hand, contemporary monovision techniques use premium IOLs, either in both eyes or at least in the non-dominant one. ${ }^{11-13}$ Contemporary monovision is supposed to offer the advantages of multifocal IOLs without the unwanted dysphotopic phenomena that are frequently encountered following their bilateral implantation.

The Restor IOL (Alcon Research, Fort Worth, TX, USA) offers an additional refractive power of $+2.50 \mathrm{D}$ for near vision. It has an apodized diffractive aspherical surface, with a central refractive zone. It is a bifocal, blue-filtered intraocular lens, which has the ability to enhance visual capacity at $53 \mathrm{~cm}$ for near targets. ${ }^{14,15}$ The Panoptix IOL (Alcon Research, Fort Worth, TX, USA) is a trifocal, non-apodized, diffractive IOL of an acrylate/methacrylate copolymer. Besides near at $40 \mathrm{~cm}(+3.20 \mathrm{D})$ and distance vision $(>4$ meters), it has the ability to enhance intermediate distance vision at $60 \mathrm{~cm}\left(+2.20\right.$ D) ${ }^{16,17}$ Both Panoptix and Restor have been bilaterally implanted in thousands of cataract patients or used in monovision approaches either with monofocal or with other premium IOLs. ${ }^{5,12,16,18,19}$

Within this context, the primary objective of this study was to compare the efficacy of premium monovision with Restor and Panoptix, against other prevalent monovision techniques and against bilateral Panoptix implantation in a sample of patients following cataract surgery.

\section{Methods}

\section{Setting}

This was a prospective, comparative, non-randomized, clinic-based trial. Study protocol adhered to the tenets of the Helsinki Declaration and written informed consent was obtained by all participants. The Ethics committee of the University Hospital of Alexandroupolis (UHA) approved the protocol. The study was conducted at the UHA, Greece, between November 2020 and June 2021.

\section{Participants}

Participants were recruited from the Cataract Service of the UHA in a consecutive-if-eligible basis. Eligibility criteria included diagnosis of senile cataract with stage 2 nuclear opalescence according to the Lens Opacities Classification System III (LOCS-3) grading scale. Four study groups were formed according to the procedure and the IOL technology that was used: 1) Monovision Group (MoG), 2) Multifocal Lens Group (MfG), 3) Hybrid Monovision Group (HmG), and, 4) Premium Monovision Group (PmG). Exclusion criteria for all participants included manifest refractive astigmatism $>1.00 \mathrm{D}$, reports of headaches and/or eyestrain associated with visual activities and positive, pathologic ocular cover test (for distance and near) and/or the Mallett's disparity test (for distance and near) and the double Maddox rod test, endothelial cell count less than $1900 / \mathrm{mm}^{2}$, glaucoma, intraocular pressure-lowering medications, former incisional surgery, former diagnosis of corneal disease, former diagnosis of fundus disease, diabetes, autoimmune, or mental diseases. For premium IOL implantations, mesopic pupil diameters below $5 \mathrm{~mm}$, chord-mu (angle kappa) below $0.4 \mathrm{~mm}$, and coma below $0.32 \mu \mathrm{m}$ were included, as derived from the Topolyzer Vario (Alcon, Fort Worth, TX). ${ }^{20}$

\section{Surgical Technique}

All operations were performed by the same surgeon (G.L.) in a consistent way using the Centurion Vision System platform and the Verion Image Guided System (Alcon, Fort Worth, TX). Pupils were dilated with Tropicamide 0.5\% (Tropixal, Demo, Greece) and phenylephrine hydrochloride 5\% (Phenylephrine, Cooper, Greece). Periorbital skin and the lids were cleaned and the conjuctival cul-de-sac was irrigated with povidone-iodine (Betadine). Patients received topical 
anesthesia with proparacaine hydrochloride $0.5 \%$ drops ( 3 drops prior to surgery). All premium implantations were digitally guided to ensure optimal outcomes. Digital guided capsulorhexis was set at $5.0 \mathrm{~mm}$ based on the visual axis.

For MoG participants, the foldable hydrophilic acrylic intraocular lens SN60WF (Alcon, Greece) was inserted in the capsular bag, targeting $-0.50 \mathrm{D}$ in the dominant eye and $-1.25 \mathrm{D}$ myopia in the non-dominant one, as described before. ${ }^{10}$ MfG patients received bilaterally the Panoptix IOL targeting emmetropia in both eyes. HmG patients received the SN60WF in the dominant eye and the Panoptix in the non-dominant one targeting emmetropia in both eyes. Finally, the PmG participants received the Restor $+2.50 \mathrm{D}$ in the dominant eye and the Panoptix IOL in the non-dominant one, targeting emmetropia in both eyes. In all cases, the non-dominant eye was initially operated, followed by the dominant one within a time window of maximum 6 months. The same postoperative regimen was prescribed to all patients, which included fixed combination of tobramycin $0.3 \%$ and dexamethasone $0.1 \%$ (FCTD), (Tobradex, Alcon, Greece) six times daily, and Sodium Hyaluronate $0.1 \%$ (Hylocomod, Pharmex, Greece) gradually tapered in a month.

\section{Data Collection}

The entire preoperative and postoperative assessment was done by the same ophthalmologist with no direct involvement in the study. The following clinical parameters were evaluated binocularly: 1) Uncorrected Distance Visual Acuity (UDVA) using the Greek version of the Early Treatment Diabetic Retinopathy Study Chart (ETDRS) at 4 meters distance, ${ }^{21}$ 2) Uncorrected Reading Acuity at $60 \mathrm{~cm}$ (UIRA) and at $40 \mathrm{~cm}$ (UNRA), and 3) Uncorrected Critical Print Size at $60 \mathrm{~cm}$ (UICPS) and at $40 \mathrm{~cm}$ (UNCPS). ${ }^{22}$ After the UDVA evaluation, all near and, then, intermediate vision parameters were obtained for all patients using the Democritus Digital Acuity Reading Test (DDART), which is based on the Greek version of MNREAD. ${ }^{23}$ Contrast sensitivity was evaluated with the Pelli-Robson test at $85 \mathrm{~cd} / \mathrm{m}^{2}$ background light.

Vision-related functional impairment was assessed using the prevalent Visual Function Index-14 (VF-14) instrument. Further to the total VF-14 score assessment, two additional scores were calculated: 1) Near vision VF score (VF14-NV) derived from items that assessed the perceived difficulty in near vision activities (items 1, 2, 3, 7, 8, 9 and 11) and, 2) Distance vision VF score (VF14-DV) derived from items that assessed the perceived difficulty of distance vision activities (items 4, 5, 6, 10, 12, 13 and 14). ${ }^{24,25}$ Dysphotopsia was evaluated with two direct 4-scale, Likert-type questions (1: Always, 2: Most of the times, 3: Sometimes, 4: Never) pertaining on the subjective perception of glare and unwanted shadows. ${ }^{10}$ The need for spectacles (ie spectacle dependence) was also evaluated for both distance [(SpectacleFree Patient for Distance Vision Activities (SFP-DV)] and near (Spectacle-Free Patient for Near Vision Activities (SFPNV)] vision by two direct 4-scale Likert-type questions (1: Always, 2: Most of the times, 3: Sometimes, 4: Never). ${ }^{10}$ All parameters were obtained 6 months following the operation of the second eye.

\section{Statistical Analysis}

An a priori power analysis was performed. For an effect size of 0.33 of the relative efficacy of the 4 different surgical procedures, 108 participants would be required in total for the study to have a power of 0.8 at the significance level of $0.05 .^{26,27}$ Distribution of measured data was tested with Kolmogorov-Smirnov test. For the normally distributed data, between-group comparisons were made using one-way ANOVA, while the non-normally distributed data were assessed with Kruskal-Wallis tests. Postoperative comparison of SFP clinical parameters among the groups were evaluated using Cochran's Q test and Mc Nemar's test after grouping the answers 1: always, 2: most of the times, 3: sometimes. P-values lower than 0.05 were considered statistically significant. All statistical analyses were performed with the MedCalc version 14.8.1 (MedCalc Software, Mariakerke, Belgium).

Regression analysis of our data allowed the construction of a mathematical model that calculated the relative efficacy of each surgical intervention based on the impact of each measured clinical parameter on the overall perceived difficulty which derived by the VF-14 score. The efficacy $f_{i}$ of the surgical intervention of each clinical parameter $x_{i}$ is derived by the following equation:

$$
f_{i}(x)=\frac{a_{i}}{q_{i}}\left(\frac{1}{1+e^{-b_{i}\left(x-x_{0}\right)}}-\frac{1}{1+e^{x_{0} b_{i}}}\right), x \in[0,100]
$$


where $x_{0}, a_{i}, b_{i}$ are constants that will obtain values in the Results section, and $q_{i}=\frac{1}{1+e^{-b_{i}\left(100-x_{0}\right)}}$. All measured clinical parameters were included in the model and contributed to the overall relative efficacy $f_{\text {all }}$, according to the equation:

$$
f_{\text {all }}(x)=\frac{1}{A} \sum_{i} f_{i}(x)
$$

where $A=\sum_{i} f_{i}(x=100)$

\section{Results}

A total of 120 participants [64 men and 56 women, $58.3 \pm 8.7$ years] were recruited and populated equally the study groups. All of them had uneventful operations and presented postoperative manifest refractive astigmatism below 0.50 $\mathrm{D}$ to ensure that astigmatism will not interfere with our outcomes. Detailed demographic data and preoperative Best Spectacle-Corrected Visual Acuity (BSCVA) are presented in Table 1. Non-significant differences were detected in the age $(\mathrm{p}=0.12)$ and BSCVA $(\mathrm{p}=0.18)$ among the groups.

Postoperative UDVA was significantly better than preoperative BSCVA for all study groups (all $p<0.01$ ). Regarding postoperative comparisons among study groups, non-significant differences could be detected in UDVA ( $\mathrm{p}=0.12)$, and UIRA ( $p=0.24)$. On the other hand, significant differences could be detected in UICPS $(p=0.04)$, UNRA $(p=0.02)$ and UNCPS ( $p=0.01$ ). MfG group presented significantly more dysphotopic phenomena (glare and shadows) followed by the PmG group ( $p=0.04$ and $p=0.02$, respectively). However, non-significant differences could be detected in contrast sensitivity $(\mathrm{p}>0.05)$. All comparisons are presented in Table 2 .

Spectacle dependence and VF-14 scores are also presented in Table 2. PmG participants demonstrated significant better total VF-14 and VF14-DV scores $(\mathrm{p}=0.04$ and $\mathrm{p}=0.01$, respectively), while MfG participants presented better VF14-NV scores $(\mathrm{p}=0.01)$. On the other hand, more PmG participants were totally spectacle-free both for near and distance vision activities [SFP (total): PmG: 83.33\%, HmG: 70\%, MfG: 76.67\% and MoG: 26.67\%].

The relative efficacy $f_{i}$ for each of the aforementioned clinical parameters $x_{i}$ was calculated according to Eq. (1). The value of $x_{0}$ in the relative efficacy $f_{i}$ was set to 47 for all parameters $x_{i}$, while the actual values of constants $a_{i}$ and $b_{i}$, as they derive from regression analysis and curve modeling for each clinical parameter $x_{i}$ (Figure 1), are presented in Table 3.

In accordance with our model, the relative efficacy $f_{i}$ of all clinical parameters in all surgical procedures is presented in Table 4. The overall relative efficacy $f_{\text {all }}$ is shown in Figure 2 for all four surgical procedures. Premium monovision demonstrated the highest relative efficacy with $92.18 \%$ followed by bilateral trifocal implantation (88.49\%), hybrid monovision (87.89\%), and monovision $(70.78 \%)$.

\section{Discussion}

In this study, we compared the efficacy of premium monovision against other prevalent monovision techniques and against the bilateral trifocal implantation. Premium monovision is a contemporary "mix-and-match" surgical approach that combines the benefits of two different premium IOLs in an attempt to provide optimal visual acuity at all distances,

Table I Demographic and Preoperative Data for Study Participants

\begin{tabular}{|l|c|c|c|c|}
\hline Study Group & $\mathbf{N}$ & Age (Years) (Mean \pm SD) & LOCS & BSCVA (logMAR) (Mean \pm SD) \\
\hline MoG & 30 & $57.4 \pm 7.4$ & Stage 2 & $0.62 \pm 0.07$ \\
\hline MfG & 30 & $60.3 \pm 8.3$ & Stage 2 & $0.55 \pm 0.11$ \\
\hline HmG & 30 & $57.0 \pm 8.8$ & Stage 2 & $0.54 \pm 0.09$ \\
\hline PmG & 30 & $57.9 \pm 9.3$ & Stage 2 & $0.60 \pm 0.06$ \\
\hline P-values & NA & 0.12 & NA & 0.18 \\
\hline
\end{tabular}

Abbreviations: BSCVA, best spectacle-corrected visual acuity (logMAR); HmG, Hybrid Monovision Group; LOCS, Lens Opacities Classification System; MfG, Multifocal Lens Group; MoG, Monovision Group; N, number of patients; NA, not applicable; PmG, Premium Monovision Group; SD, standard deviation. 
Table 2 Postoperative Comparisons of All Measured Clinical Parameters

\begin{tabular}{|c|c|c|c|c|c|c|c|c|}
\hline \multirow{3}{*}{$\begin{array}{l}\text { Clinical Parameters } \\
\text { UDVA (logMAR) }\end{array}$} & MoG & MfG & HmG & PmG & \multirow{2}{*}{\multicolumn{4}{|c|}{ p-values }} \\
\hline & \multicolumn{4}{|c|}{ Mean \pm SD } & & & & \\
\hline & $0.04 \pm 0.06$ & $0.03 \pm 0.03$ & $0.05 \pm 0.04$ & $0.03 \pm 0.02$ & \multicolumn{4}{|c|}{0.12} \\
\hline UIRA (logMAR) & $0.08 \pm 0.05$ & $0.05 \pm 0.04$ & $0.06 \pm 0.03$ & $0.05 \pm 0.02$ & \multicolumn{4}{|c|}{0.24} \\
\hline UICPS (logMAR) & $0.24 \pm 0.08$ & $0.13 \pm 0.04$ & $0.18 \pm 0.09$ & $0.11 \pm 0.04$ & \multicolumn{4}{|c|}{$0.04 *$} \\
\hline UNRA (logMAR) & $0.23 \pm 0.09$ & $0.05 \pm 0.08$ & $0.08 \pm 0.07$ & $0.07 \pm 0.06$ & \multicolumn{4}{|c|}{$0.02 *$} \\
\hline UNCPS (logMAR) & $0.34 \pm 0.08$ & $0.14 \pm 0.10$ & $0.20 \pm 0.09$ & $0.17 \pm 0.11$ & \multicolumn{4}{|c|}{$0.01 *$} \\
\hline CS & $1.74 \pm 0.16$ & $1.61 \pm 0.22$ & $1.67 \pm 0.23$ & $1.63 \pm 0.19$ & \multicolumn{4}{|c|}{0.13} \\
\hline Glare & $0.24 \pm 0.35$ & $1.41 \pm 0.99$ & $0.78 \pm 0.67$ & $0.85 \pm 0.45$ & \multicolumn{4}{|c|}{$0.04 *$} \\
\hline Shadows & $0.55 \pm 0.27$ & $1.54 \pm 0.75$ & $0.87 \pm 0.85$ & $0.94 \pm 1.01$ & \multicolumn{4}{|c|}{$0.02 *$} \\
\hline VF-14 score & $85.84 \pm 10.14$ & $88.23 \pm 9.13$ & $87.39 \pm 11.14$ & $92.44 \pm 9.45$ & \multicolumn{4}{|c|}{$0.04 *$} \\
\hline VFI4-NV & $81.77 \pm 9.32$ & $92.37 \pm 9.37$ & $83.85 \pm 12.58$ & $90.69 \pm 10.08$ & \multicolumn{4}{|c|}{$0.01 *$} \\
\hline VFI4-DV & $89.92 \pm 9.25$ & $85.08 \pm 10.07$ & $90.93 \pm 11.74$ & $94.20 \pm 8.63$ & \multicolumn{4}{|c|}{$0.01 *$} \\
\hline \multirow[t]{5}{*}{ SFP-NV (\%) } & \multirow[t]{5}{*}{26.66} & \multirow[t]{5}{*}{90.00} & \multirow[t]{5}{*}{76.67} & \multirow[t]{5}{*}{86.67} & & MoG & MfG & $\mathrm{HmG}$ \\
\hline & & & & & MfG & $<0.001$ & & \\
\hline & & & & & $\mathrm{HmG}$ & $<0.001$ & 0.0625 & \\
\hline & & & & & PmG & $<0.001$ & 1.000 & 0.125 \\
\hline & & & & & \multicolumn{4}{|c|}{$<0.001$} \\
\hline \multirow[t]{5}{*}{ SFP-DV (\%) } & \multirow[t]{5}{*}{86.67} & \multirow[t]{5}{*}{83.33} & \multirow[t]{5}{*}{83.33} & \multirow[t]{5}{*}{93.33} & & MoG & MfG & $\mathrm{HmG}$ \\
\hline & & & & & MfG & 0.50 & & \\
\hline & & & & & $\mathrm{HmG}$ & 0.50 & 1.000 & \\
\hline & & & & & PmG & $<0.001$ & 0.125 & 0.125 \\
\hline & & & & & \multicolumn{4}{|c|}{0.063} \\
\hline \multirow[t]{5}{*}{ SFP (total) (\%) } & \multirow[t]{5}{*}{26.67} & \multirow[t]{5}{*}{76.67} & \multirow[t]{5}{*}{70.00} & \multirow[t]{5}{*}{83.33} & & MoG & MfG & $\mathrm{HmG}$ \\
\hline & & & & & MfG & $<0.001$ & & \\
\hline & & & & & $\mathrm{HmG}$ & $<0.001$ & 0.50 & \\
\hline & & & & & PmG & $<0.001$ & 0.50 & 0.13 \\
\hline & & & & & & & & \\
\hline
\end{tabular}

Note: $*_{p}<0.05$

Abbreviations: UDVA, uncorrected distance visual acuity; UIRA, uncorrected intermediate reading acuity (at $60 \mathrm{~cm}$ ); UICPS, uncorrected intermediate critical print size (at $60 \mathrm{~cm}$ ); UNRA, uncorrected near reading acuity (at $40 \mathrm{~cm}$ ); UNCPS, uncorrected near critical print size (at $40 \mathrm{~cm}$ ); CS, contrast sensitivity; HmG, Hybrid Monovision Group; MfG, Multifocal Lens Group; MoG, Monovision Group; PmG, Premium Monovision Group; SD, standard deviation; SFP-NV, spectacle-free patients for near vision activities; SFP-DV, spectacle-free patients for distance vision activities; SFP (total), spectacle-free patients for all distances; VF-I4, Visual Function Index I4 questionnaire, VFI4-NV, Visual Function Index 14 questionnaire Near Vision Score; VFI4-DV, Visual Function Index I4 questionnaire Distance Vision Score.

minimizing the drawbacks of bilateral multifocal corrections. ${ }^{28,29}$ In our case, we used two premium IOLs with completely different characteristics: the hybrid bifocal Restor $+2.5 \mathrm{D}$ implanted in the dominant eye and the diffractive trifocal Panoptix implanted in the non-dominant one. Restor's central refractive zone (ActiveFocus) is supposed to provide optimal distance visual acuity, which is comparable to a monofocal IOL. Light distribution at the distant focal 


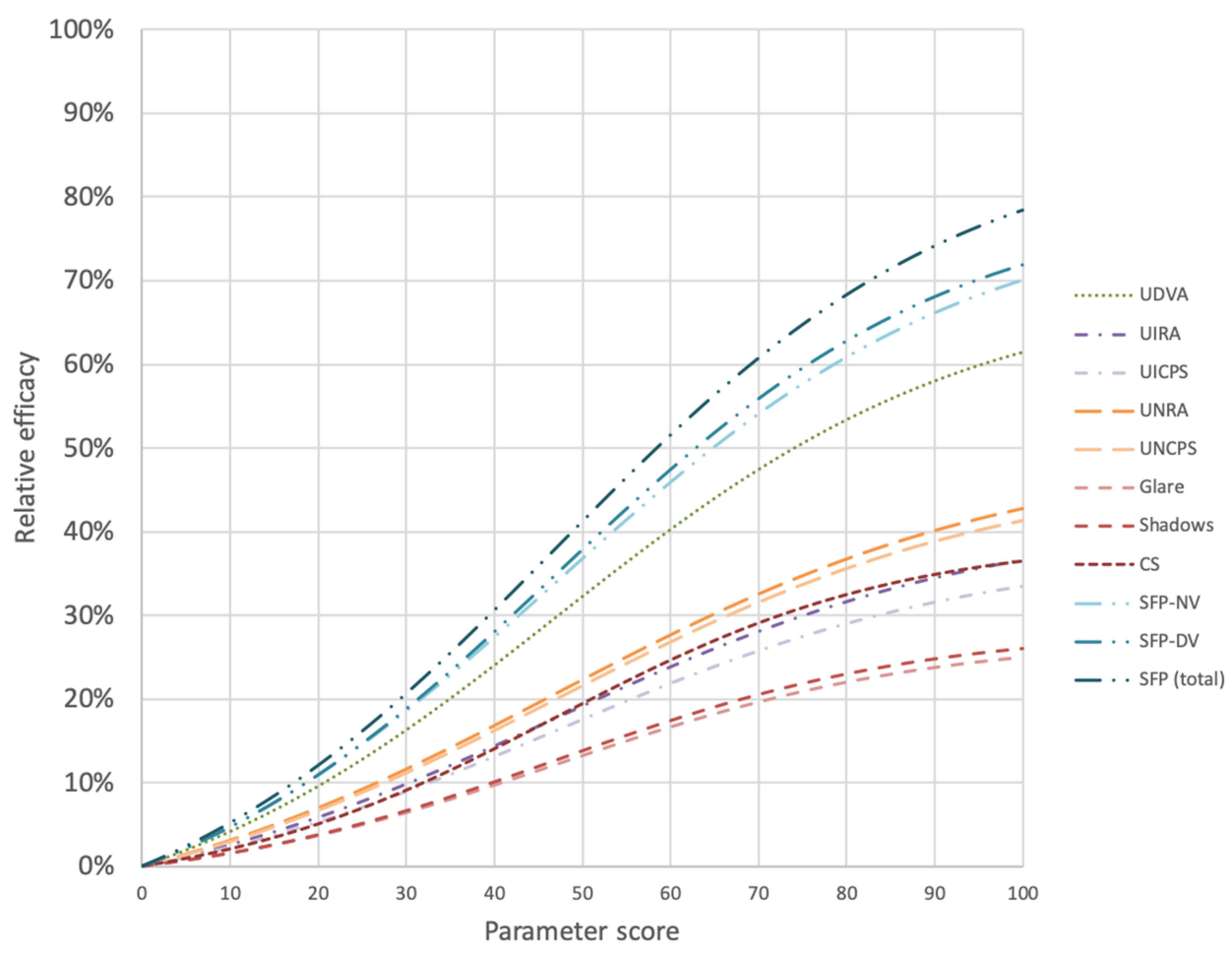

Figure I Relative efficacy as a function of each parameter score.

Abbreviations: UDVA, uncorrected distance visual acuity; UIRA, uncorrected intermediate reading acuity (at $60 \mathrm{~cm}$ ); UICPS, uncorrected intermediate critical print size (at $60 \mathrm{~cm}$ ); UNRA, uncorrected near reading acuity (at $40 \mathrm{~cm}$ ); UNCPS, uncorrected critical print size (at $40 \mathrm{~cm}$ ); CS, contrast sensitivity; SFP (total), spectacle-free patient (all distances); SFP-DV, spectacle-free patient for distance vision activities; SFP-NV, spectacle-free patient for near vision activities; VF-I4, Visual Function Index questionnaire.

point of Restor is $69.4 \%$, quite close to the $87 \%$ of the monofocal SN60WF that shares the same manufacturing characteristics. Moreover, Restor distributes $18 \%$ of the available light at $53 \mathrm{~cm}$ providing an intermediate focal point. ${ }^{15}$ On the other hand, Panoptix is a full diffractive IOL that has a complex light distribution technology (Enlighten), which provides $44 \%$ of the available light at the distant focal point, $22 \%$ at $60 \mathrm{~cm}$ (intermediate focal point) and $22 \%$ at $40 \mathrm{~cm}$ (near focal point). ${ }^{30}$ In theory, premium monovision with Restor and Panoptix offers highquality distance vision even in low-light conditions, due to the high light distribution of the Restor at the distant focal point, and near-perfect intermediate and near vision from the three focal points that are close enough to simulate the prepresbyopic functionality of the eye.

Bilateral myopic mini-monovision has been described before by our group. ${ }^{10}$ It is associated with high satisfaction rates, since it is well tolerated, it does not compromise contrast sensitivity or stereopsis and offers spectacle-free distance and intermediate vision in the majority of patients.

Hybrid monovision combines a monofocal IOL in the dominant eye and a premium lens in the non-dominant one. ${ }^{13}$ It offers spectacle-free, distance vision with minimal optical phenomena since a monofocal IOL is implanted in the dominant eye, and two monocular focal points at 40 and $60 \mathrm{~cm}$.

Bilateral trifocal implantation has been extensively studied by former researchers. It produces excellent results in terms of spectacle independence, but it suffers from reduced efficacy and optical phenomena especially in low-light conditions. ${ }^{31-34}$

We attempted to quantify the efficacy of each surgical intervention with the construction of the dependent variable "relative efficacy" for all measured clinical parameters that was based on the total VF-14 score. However, further to reading acuity, near and intermediate vision was evaluated with critical print size, as well, since the later is the smallest print size that can be read in maximal speed, being thus a more accurate index of reading capacity. ${ }^{22,35}$ For the accurate mathematical construction of the relative efficacy, we used regression analysis and curve modeling, which revealed the relative impact of each parameter on the dependent variable and allowed its overall estimation. 
Table 3 Values of Constants $a_{i}$ and $b_{i}$

\begin{tabular}{|l|c|c|}
\hline \multirow{2}{*}{ Clinical Parameters } & \multicolumn{2}{|c|}{ Constants' Values } \\
\cline { 2 - 3 } & $a_{\boldsymbol{i}}$ & $\boldsymbol{b}_{\boldsymbol{i}}$ \\
\hline UDVA & 0.71 & 0.042 \\
\hline UIRA & 0.43 & 0.040 \\
\hline UICPS & 0.39 & 0.041 \\
\hline UNRA & 0.51 & 0.038 \\
\hline UNCPS & 0.49 & 0.039 \\
\hline Glare & 0.28 & 0.047 \\
\hline Shadows & 0.29 & 0.048 \\
\hline CS & 0.40 & 0.051 \\
\hline SFP-NV & 0.81 & 0.042 \\
\hline SFP-DV & 0.82 & 0.044 \\
\hline SFP (total) & 0.90 & 0.043 \\
\hline
\end{tabular}

Abbreviations: UDVA, uncorrected distance visual acuity; UIRA, uncorrected intermediate reading acuity (at $60 \mathrm{~cm}$ ); UICPS, uncorrected intermediate critical print size (at $60 \mathrm{~cm}$ ); UNRA, uncorrected near reading acuity (at $40 \mathrm{~cm}$ ); UNCPS, uncorrected near critical print size (at $40 \mathrm{~cm}$ ); CS, contrast sensitivity; SFP-NV, spectacle-free patients for near vision activities; SFP-DV, spectacle-free patients for distance vision activities; SFP (total), spectacle-free patients for all distances.

Table 4 Relative Efficacy of Each Intervention

\begin{tabular}{|c|c|c|c|c|c|}
\hline \multirow[t]{2}{*}{ Clinical Parameters } & \multicolumn{4}{|c|}{ Surgical Intervention (\%) } & \multirow[t]{2}{*}{ Optimal (\%) } \\
\hline & MoG & MfG & HmG & PmG & \\
\hline UDVA & 96 & 97 & 95 & 97 & 100 \\
\hline UIRA & 92 & 95 & 94 & 95 & 100 \\
\hline UICPS & 76 & 87 & 82 & 89 & 100 \\
\hline UNRA & 77 & 95 & 92 & 93 & 100 \\
\hline UNCPS & 66 & 86 & 80 & 83 & 100 \\
\hline Glare & 92 & 53 & 74 & 72 & 100 \\
\hline Shadows & 82 & 49 & 71 & 69 & 100 \\
\hline CS & 87 & 80 & 83 & 81 & 100 \\
\hline SFP-NV & 27 & 90 & 77 & 87 & 100 \\
\hline SFP-DV & 87 & 83 & 83 & 93 & 100 \\
\hline SFP (total) & 27 & 77 & 70 & 83 & 100 \\
\hline Relative Efficacy & 70.78 & 88.49 & 87.89 & 92.18 & 100 \\
\hline
\end{tabular}

Abbreviations: UDVA, uncorrected distance visual acuity; UIRA, uncorrected intermediate reading acuity (at $60 \mathrm{~cm}$ ), UICPS, uncorrected intermediate critical print size (at $60 \mathrm{~cm}$ ); UNRA, uncorrected near reading acuity (at $40 \mathrm{~cm}$ ); UNCPS, uncorrected near critical print size (at $40 \mathrm{~cm}$ ); CS, contrast sensitivity; HmG, Hybrid Monovision Group; MfG, Multifocal Lens Group; MoG, Monovision Group; PmG, Premium Monovision Group; SFPNV, spectacle-free patient for near vision activities; SFP-DV, spectacle-free patient for distance vision activities; SFP (total), spectacle-free patient (all distances). 


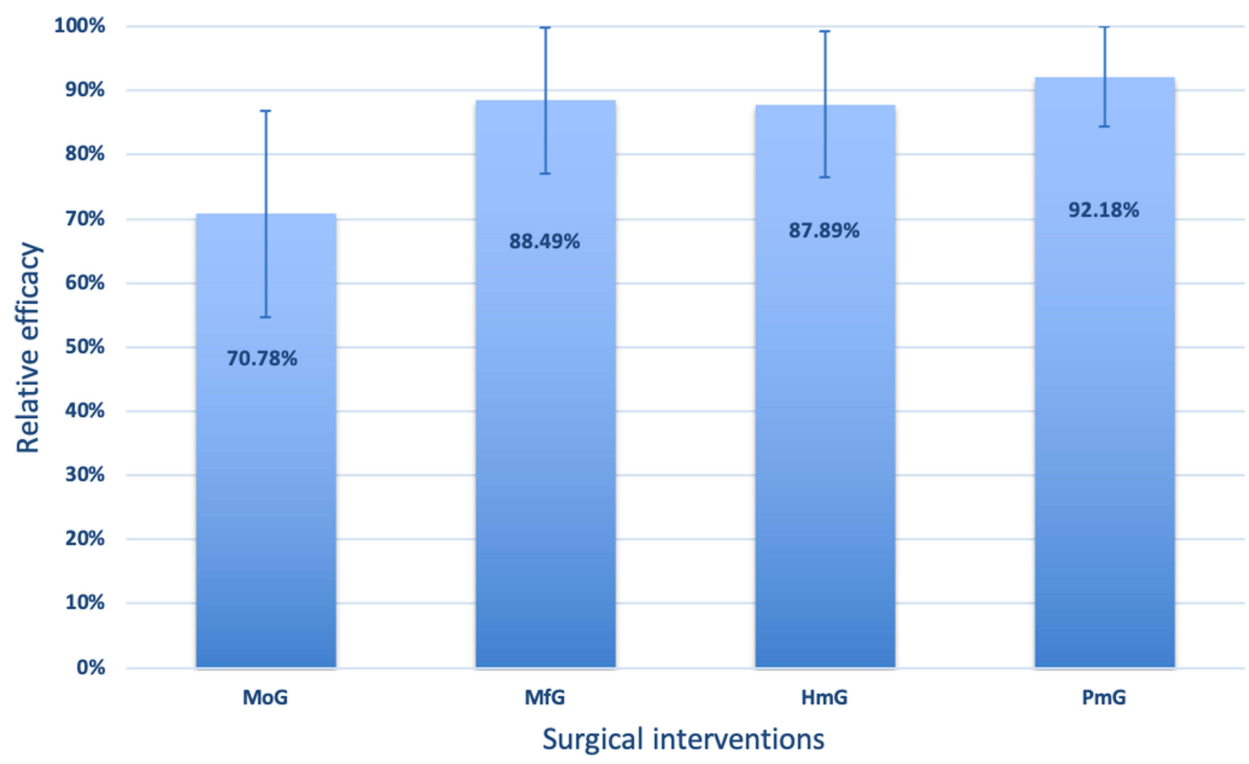

Figure 2 Relative efficacy of each intervention.

Abbreviations: HmG, Hybrid Monovision Group; MfG, Multifocal Lens Group; MoG, Monovision Group; PmG, Premium Monovision Group.

Interesting conclusions derive from our model. As presented in Figure 1,1) all clinical parameters have non-linear correlation with relative efficacy, 2) they contribute unevenly to the dependent variable, and 3) they demonstrate different growth rate. However, our model is consistent with clinical experience. For example, the SFP (total) parameter contributes the most to relative efficacy $\left(a_{S F P}\right.$ (Total) $\left.=0.90\right)$ suggesting that a spectacle-free vision for all distances is the ultimate objective for patient satisfaction. On the other hand, the prevalent clinical parameters of distance, intermediate and near visual acuity that are supposed to objectively reflect the visual capacity of the patient, contribute much less to the variable relative efficacy $\left(a_{U D V A}=0.71, a_{U I V A}=0.43, a_{U N V A}=0.51\right.$, respectively). This finding suggests that presbyopic patients perceive the surgical intervention's successful or less successful outcome subjectively and according to their individual needs and wants. Another interesting finding from our curve modeling was that dysphotopsia's impact on the relative efficacy was minimal $\left(a_{\text {Glare }}=0.28, a_{\text {Shadows }}=0.29\right)$. A possible explanation to this finding is that, in our department, we have very strict criteria in patient selection for pseudophakic presbyopic corrections; therefore, we rarely encounter cases with severe dysphotopic phenomena (that would excessively contribute to the dependent variable), since patients with large mesopic pupil diameters, high centroid shifts, or night-shift working mandates are a priori excluded from multifocal implantations.

In accordance with the above, our outcomes indicated an overall superiority of premium monovision against all other surgical approaches that we used, as expressed by the relative efficacy scores. Specifically, the premium monovision participants together with the bilateral multifocal group ones demonstrated: 1) the best distance vision and 2) the best intermediate vision. Moreover, PmG participants demonstrated the best overall spectacle independence. On the other hand, myopic monovision group presented the lowest optical phenomena and the best contrast sensitivity, while the bilateral multifocal group presented the best near vision capacity and the second overall relative efficacy score.

Despite different methods used in former published reports, our outcomes are in accordance with the literature. Several studies concluded that both monovision techniques and the implantation of multifocal IOLs offer satisfactory distance visual outcomes, while the near visual acuity seems to be better in multifocal patients. ${ }^{10,36}$ Distance vision spectacle independence in monovision patients is comparable with the multifocal IOL implantation, which is associated with more dysphotopic phenomena. ${ }^{10,37}$ However, when examining near and intermediate vision acuity with a CPS index, monofocal monovision patients are most likely to present average scores since CPS is a very demanding index of reading capacity. 
Certain limitations of our study should be taken into consideration when interpreting our outcomes. No randomization in the population of study groups was attempted since in our Department the selection of the presbyopic surgical intervention depends heavily on the patient's subjective needs and wants and not only on his/her clinical data. Therefore, our relative efficacy model is valid only when strict selection criteria are applied prior to multifocal implantations. Moreover, the assessment of the relative efficacy of each intervention was based on a specific set of clinical parameters. Possibly, a different set of clinical parameters might produce slightly different outcomes. On the other hand, although the VF-14 Questionnaire is a well-known validated instrument, its near (VF14-NV) and distance (VF14-DV) vision subdomains have not been validated. Therefore, interpretation of these subdomains should be done with caution.

\section{Conclusions}

To our knowledge, this is the first study to introduce a mathematical model that evaluates the relative efficacy of prevalent pseudophakic presbyopic corrections. All aforementioned techniques are viable options in addressing the postoperative loss of accommodation in lens extraction surgery. Each technique has advantages and drawbacks that should be taken into consideration before selecting the most compatible approach to the patient's needs and wants. Nevertheless, premium monovision seems to demonstrate impressive spectacle-free postoperative visual capacity at all distances with minimal dysphotopic phenomena; therefore, it should be among the primary options in presbyopia correction.

\section{Abbreviations}

BSCVA, best spectacle-corrected visual acuity; UDVA, uncorrected distance visual acuity; UICPS, critical print size at $60 \mathrm{~cm}$; UIRA, uncorrected reading acuity at $60 \mathrm{~cm}$; UNCPS, critical print size at $40 \mathrm{~cm}$; UNRA, uncorrected reading acuity at $40 \mathrm{~cm}$; D, Diopters; DDART, Democritus Digital Acuity Reading Test; ETDRS, Early Treatment Diabetic Retinopathy Study Chart; FCTD, fixed combination of tobramycin $0.3 \%$ and dexamethasone $0.1 \%$; HmG, Hybrid Monovision Group; IOL, intraocular lens; LOCS-3, Lens Opacities Classification System III grading scale; MfG, Multifocal Lens Group; MoG, Monovision Group; PmG, Premium Monovision Group; SFP-DV, Spectacle-Free Patient for Distance Vision Activities; SFP-NV, Spectacle-Free Patient for Near Vision Activities; UHA, University Hospital of Alexandroupolis; VF-14, total Visual Function Index-14 score; VF14-DV, Distance vision Visual Function Index-14 score; VF14-NV, Near vision Visual Function Index-14 score.

\section{Data Sharing Statement}

De-identified data are available in print form for 1 year following the conclusion of the study.

\section{Ethics Approval and Consent to Participate}

This research followed the tenets of the Declaration of Helsinki. Approval was obtained by the Ethics committee of the University Hospital of Alexandroupolis. All participants provided written informed consent.

\section{Author Contributions}

GL conceived, designed, and supervised the study, as well as wrote the main manuscript and critically revised it. EKP contributed to data collection/data analysis/interpretation of data/statistical analysis, and critically revised the manuscript. AP and PN contributed to data collection and critically revised the manuscript. KD conributed to data analysis/ interpretation of data/statistical analysis, and critically revised the manuscript. IF, AK, and DD contributed to the data analysis/interpretation of data, and critically revised the manuscript. In general, all authors made substantial contributions to conception and design, acquisition of data, or analysis and interpretation of data; took part in drafting the article or revising it critically for important intellectual content; agreed to submit to the current journal; gave final approval of the version to be published; and agree to be accountable for all aspects of the work.

\section{Disclosure}

The authors declare that they have no competing interests in this work. 


\section{References}

1. Sharma B, Abell RG, Arora T, Antony T, Vajpayee RB. Techniques of anterior capsulotomy in cataract surgery. Indian J Ophthalmol. 2019;67 (4):450-460. doi:10.4103/ijo.IJO_1728_18

2. Gil-Cazorla R, Shah S, Naroo SA. A review of the surgical options for the correction of presbyopia. Br J Ophthalmol. 2016;100(1):62-70. doi:10.1136/bjophthalmol-2015-306663

3. Mahrous A, Ciralsky JP, Lai EC. Revisiting monovision for presbyopia. Curr Opin Ophthalmol. 2018;29(4):313-317. doi:10.1097/ ICU.0000000000000487

4. Xiao J, Jiang C, Zhang M. Pseudophakic monovision is an important surgical approach to being spectacle-free. Indian J Ophthalmol. 2011;59 (6):481-485. doi:10.4103/0301-4738.86318

5. Zhang F, Sugar A, Jacobsen G, Collins M. Visual function and spectacle Independence after cataract surgery: bilateral diffractive multifocal intraocular lenses versus monovision pseudophakia. J Cataract Refract Surg. 2011;37(5):853-858. doi:10.1016/j.jcrs.2010.12.041

6. Zettl S, Reiß S, Terwee T, Guthoff RF, Beck R, Stachs O. Effect of pseudophacic mini-monovision as an option for Independence of spectacles in everyday life. Klin Monbl Augenheilkd. 2014;231(12):1196-1202. doi:10.1055/s-0034-1383367

7. Labiris G, Toli A, Perente A, Ntonti P, Kozobolis VP. A systematic review of pseudophakic monovision for presbyopia correction. Int J Ophthalmol. 2017;10(6):992-1000. doi:10.18240/ijo.2017.06.24

8. Greenstein S, Pineda R 2nd. The quest for spectacle independence: a comparison of multifocal intraocular lens implants and pseudophakic monovision for patients with presbyopia. Semin Ophthalmol. 2017;32(1):111-115. doi:10.1080/08820538.2016.1228400

9. Goldberg DG, Goldberg MH, Shah R, Meagher JN, Ailani H. Pseudophakic mini-monovision: high patient satisfaction, reduced spectacle dependence, and low cost. BMC Ophthalmol. 2018;18(1):293. doi:10.1186/s12886-018-0963-3

10. Labiris G, Giarmoukakis A, Patsiamanidi M, Papadopoulos Z, Kozobolis VP. Mini-monovision versus multifocal intraocular lens implantation. $J$ Cataract Refract Surg. 2015;41(1):53-57. doi:10.1016/j.jcrs.2014.06.015

11. Chang JSM, Liu SCT, Ng JCM, Ma PL. Monovision with a bifocal diffractive multifocal intraocular lens in presbyopic patients: a prospective, observational case series. Am J Ophthalmol. 2020;212:105-111. doi:10.1016/j.ajo.2019.11.010

12. Ravikumar S, Bradley A, Bharadwaj S, Thibos LN. Expanding binocular depth of focus by combining monovision with diffractive bifocal intraocular lenses. J Cataract Refract Surg. 2016;42(9):1288-1296. doi:10.1016/j.jcrs.2016.04.036

13. Iida Y, Shimizu K, Ito M. Pseudophakic monovision using monofocal and multifocal intraocular lenses: hybrid monovision. $J$ Cataract Refract Surg. 2011;37(11):2001-2005. doi:10.1016/j.jcrs.2011.05.032

14. Alcon Laboratories, Inc. AcrySof IQ Restor, Product information. Available from: https://www.accessdata.fda.gov/cdrh_docs/pdf4/P040020S050d. pdf. Accessed January 13, 2022.

15. Carson D, Hill WE, Hong X, Karakelle M. Optical bench performance of AcrySof $\left({ }^{\circledR}\right)$ IQ ReSTOR $\left({ }^{\circledR}\right)$, AT LISA $\left({ }^{\circledR}\right)$ tri, and FineVision $\left({ }^{\circledR}\right)$ intraocular lenses. Clin Ophthalmol. 2014;8:2105-2113. doi:10.2147/OPTH.S66760

16. Lee S, Choi M, Xu Z, Zhao Z, Alexander E, Liu Y. Optical bench performance of a novel trifocal intraocular lens compared with a multifocal intraocular lens. Clin Ophthalmol. 2016;10:1031-1038. doi:10.2147/OPTH.S106646

17. AcrySof IQ panoptix, summary of safety and effectiveness data. Fort Worth, TX: Alcon Laboratories, Inc; 2019. Available from: https://www. accessdata.fda.gov/cdrh_docs/pdf4/P040020S087B.pdf. Accessed January 13, 2022.

18. Vilar C, Hida WT, de Medeiros AL, et al. Comparison between bilateral implantation of a trifocal intraocular lens and blended implantation of two bifocal intraocular lenses. Clin Ophthalmol. 2017;11:1393-1397. doi:10.2147/OPTH.S139909

19. Chiam PJT, Chan JH, Haider SI, Karia N, Kasaby H, Aggarwal RK. Functional vision with bilateral ReZoom and ReSTOR intraocular lenses 6 months after cataract surgery. J Cataract Refract Surg. 2007;33(12):2057-2061. doi:10.1016/j.jcrs.2007.07.029

20. Fu Y, Kou J, Chen D, et al. Influence of angle kappa and angle alpha on visual quality after implantation of multifocal intraocular lenses. J Cataract Refract Surg. 2019;45(9):1258-1264. doi:10.1016/j.jcrs.2019.04.003

21. Plainis S, Moschandreas J, Giannakopoulou T, et al. Validation of a modified ETDRS chart for European-wide use in populations that use the Cyrillic, Latin or Greek alphabet. J Optom. 2013;6(1):18-24. doi:10.1016/j.optom.2012.06.008

22. Dupps WJ, Kohnen T, Mamalis N, et al. Standardized graphs and terms for refractive surgery results. J Cataract Refract Surg. 2011;37(1):1-3. doi:10.1016/j.jcrs.2010.11.010

23. Labiris G, Panagiotopoulou EK, Chatzimichael E, Tzinava M, Mataftsi A, Delibasis K. Introduction of a digital near-vision reading test for normal and low vision adults: development and validation. Eye Vis. 2020;7(1):51. doi:10.1186/s40662-020-00216-0

24. Labiris G, Ntonti P, Patsiamanidi M, Sideroudi H, Georgantzoglou K, Kozobolis VP. Evaluation of activities of daily living following pseudophakic presbyopic correction. Eye Vis. 2017;4(1):2. doi:10.1186/s40662-016-0067-1

25. Mylona I, Aletras V, Ziakas N, Tsinopoulos I. Rasch validation of the VF-14 scale of vision-specific functioning in Greek patients. Int J Environ Res Public Health. 2021;18(8):4254. doi:10.3390/ijerph18084254

26. Cohen J. Statistical Power Analysis for the Behavioral Sciences. 2nd ed. New Jersey: Lawrence Erlbaum; 1988.

27. Becker LA. Effect Size (ES); 2000. Available from: http://www.bwgriffin.com/gsu/courses/edur9131/content/EffectSizeBecker.pdf. Accessed January 13, 2022.

28. Hütz WW, Bahner K, Röhrig B, Hengerer F. The combination of diffractive and refractive multifocal intraocular lenses to provide full visual function after cataract surgery. Eur J Ophthalmol. 2010;20(2):370-375. doi:10.1177/112067211002000217

29. Gunenc U, Celik L. Long-term experience with mixing and matching refractive array and diffractive CeeOn multifocal intraocular lenses. $J$ Refract Surg. 2008;24(3):233-242.

30. Sudhir RR, Dey A, Bhattacharrya S, Bahulayan A. AcrySof IQ panoptix intraocular lens versus extended depth of focus intraocular lens and trifocal intraocular lens: a clinical overview. Asia Pac J Ophthalmol. 2019;8(4):335-349. doi:10.1097/APO.0000000000000253

31. de Medeiros AL, de AraújoRolim AG, Motta AFP, et al. Comparison of visual outcomes after bilateral implantation of a diffractive trifocal intraocular lens and blended implantation of an extended depth of focus intraocular lens with a diffractive bifocal intraocular lens. Clin Ophthalmol. 2017;11:1911-1916. doi:10.2147/OPTH.S145945

32. Hayashi K, Sato T, Igarashi C, Yoshida M. Comparison of visual outcomes between bilateral trifocal intraocular lenses and combined bifocal intraocular lenses with different near addition. Jpn J Ophthalmol. 2019;63(6):429-436. doi:10.1007/s10384-019-00693-4 
33. Mencucci R, Favuzza E, Caporossi O, Savastano A, Rizzo S. Comparative analysis of visual outcomes, reading skills, contrast sensitivity, and patient satisfaction with two models of trifocal diffractive intraocular lenses and an extended range of vision intraocular lens. Graefes Arch Clin Exp Ophthalmol. 2018;256(10):1913-1922. doi:10.1007/s00417-018-4052-3

34. Labiris G, Ntonti P, Panagiotopoulou EK, et al. Impact of light conditions on reading ability following multifocal pseudophakic corrections. Clin Ophthalmol. 2018;12:2639-2646. doi:10.2147/OPTH.S180766

35. Calabrèse A, Cheong AM, Cheung $\mathrm{SH}$, et al. Baseline MNREAD measures for normally sighted subjects from childhood to old age. Invest Ophthalmol Vis Sci. 2016;57(8):3836-3843. doi:10.1167/iovs.16-19580

36. Wilkins MR, Allan BD, Rubin GS, et al.; Moorfields IOL Study Group. Randomized trial of multifocal intraocular lenses versus monovision after bilateral cataract surgery. Ophthalmology. 2013;120(12):2449-55.e1. doi:10.1016/j.ophtha.2013.07.048

37. Kelava L, Barić H, Bušić M, Čima I, Trkulja V. Monovision versus multifocality for presbyopia: systematic review and meta-analysis of randomized controlled trials. Adv Ther. 2017;34(8):1815-1839. doi:10.1007/s12325-017-0579-7

Clinical Ophthalmology

Dovepress

\section{Publish your work in this journal}

Clinical Ophthalmology is an international, peer-reviewed journal covering all subspecialties within ophthalmology. Key topics include: Optometry; Visual science; Pharmacology and drug therapy in eye diseases; Basic Sciences; Primary and Secondary eye care; Patient Safety and Quality of Care Improvements. This journal is indexed on PubMed Central and CAS, and is the official journal of The Society of Clinical Ophthalmology (SCO). The manuscript management system is completely online and includes a very quick and fair peer-review system, which is all easy to use. Visit http://www. dovepress.com/testimonials.php to read real quotes from published authors.

Submit your manuscript here: https://www.dovepress.com/clinical-ophthalmology-journal 